\title{
The atypical chemokine receptor D6 suppresses the development of chemically induced skin tumors
}

\author{
Robert J.B. Nibbs, ${ }^{1}$ Derek S. Gilchrist, ${ }^{1}$ Vicky King, ${ }^{1}$ Antonio Ferra, ${ }^{1}$ Steve Forrow, ${ }^{2}$ \\ Keith D. Hunter, ${ }^{3}$ and Gerard J. Graham ${ }^{1}$ \\ 'Division of Immunology, Infection and Inflammation, Glasgow Biomedical Research Centre, University of Glasgow, Glasgow, United Kingdom. \\ ${ }^{2}$ CR-UK Beatson Laboratories, The Beatson Institute for Cancer Research, Glasgow, United Kingdom. ${ }^{3}$ Glasgow Dental School, \\ University of Glasgow, Glasgow, United Kingdom.
}

\begin{abstract}
A subset of CC chemokines, acting through CC chemokine receptors (CCRs) 1 to 5 , is instrumental in shaping inflammatory responses. Recently, we and others have demonstrated that the atypical chemokine receptor D6 actively sequesters and destroys many of these proinflammatory CC chemokines. This is critical for effective resolution of inflammation in vivo. Inflammation can be protumorigenic, and proinflammatory CC chemokines have been linked with various aspects of cancer biology, yet there is scant evidence supporting a critical role for these molecules in de novo tumor formation. Here, we show that D6-deficient mice have increased susceptibility to cutaneous tumor development in response to chemical carcinogenesis protocols and, remarkably, that $D 6$ deletion is sufficient to make resistant mouse strains susceptible to invasive squamous cell carcinoma. Conversely, transgenic D6 expression in keratinocytes dampens cutaneous inflammation and can confer considerable protection from tumor formation in susceptible backgrounds. Tumor susceptibility consistently correlated with the level of recruitment of $T$ cells and mast cells, cell types known to support the development of skin tumors in mice. These data demonstrate the importance of proinflammatory CC chemokines in de novo tumorigenesis and reveal chemokine sequestration by $\mathrm{D} 6$ to be a novel and effective method of tumor suppression.
\end{abstract}

\section{Introduction}

Chemokines are multifunctional secreted peptides that play a critical role in regulating leukocyte migration. They are divided into 4 subgroups (CC, $\mathrm{CXC}, \mathrm{CX}_{3} \mathrm{C}$, and $\mathrm{XC}$ ) according to a characteristic cysteine motif (1). The $\mathrm{CC}$ family contains a subset of proinflammatory chemokines (referred to hereafter as "iCC" chemokines) including CC chemokine ligands (CCLs) 2, 3, 4, 5, $7,8,11,17$, and 22 . iCC chemokines are rapidly and transiently induced in response to damage or infection and help drive inflammation by regulating leukocyte recruitment and activity (1). Inflammation is essential for effective host defense and tissue repair, yet chronic inflammation can predispose humans to cancer and animal studies have clearly demonstrated the protumorigenic properties of inflammation (2-13). Moreover, as tumors evolve they appear to actively modulate their inflammatory infiltrate, exploiting leukocytes to aid their survival and progression: significantly, many established human tumors constitutively produce, or induce, iCC chemokines, and high expression can correlate with poor prognosis (14-16). Tumor grafting models have shown that experimental modification of proinflammatory chemokine activity can alter leukocyte recruitment, angiogenesis, and tumor growth (14), yet to our knowledge only a few reports have convincingly shown indispensable roles for

Nonstandard abbreviations used: B6, C57BL/6; BioCCL3, biotinylated CCL3; CCL, $\mathrm{CC}$ chemokine ligand; $\mathrm{CCR}, \mathrm{CC}$ chemokine receptor; $\mathrm{DAB}, 3$,3-diaminobenzidine tetrahydrochloride; DMBA, 7,12-dimethylbenz(a)anthracene; iCC, proinflammatory CC; K14, keratin 14; LEC, lymphatic EC; MC, mast cell; PMN, polymorphonucleocyte; SCC, squamous cell carcinoma; TPA, 12-O-tetradecanoyl phorbol-13-acetate. Conflict of interest: The authors have declared that no conflict of interest exists. Citation for this article: J. Clin. Invest. 117:1884-1892 (2007). doi:10.1172/JCI30068. individual components of the iCC chemokine network during de novo tumor formation (17-19).

Leukocyte responses to iCC chemokines are transduced by CC chemokine receptors (CCRs) 1 to 5 , a group of heptahelical, multichemokine-binding, $\mathrm{G}$ protein-coupled receptors (1). However, most iCC chemokines also bind to D6, a promiscuous "atypical" chemokine receptor expressed by lymphatic ECs (LECs), trophoblasts, and some leukocyte subsets (20-24). Despite close structural similarity to CCR 1 to -5 , D6 appears unable to initiate intracellular signals $(21-23,25)$. However, constitutive trafficking to and from the cell surface, in the absence of chemokine-driven desensitization, allows D6 to progressively and efficiently deplete extracellular iCC chemokines in vitro (26-28). Significantly, we and others have shown that D6 reduces iCC chemokine bioavailability in vivo to ensure appropriate resolution of inflammatory responses $(24,29-31)$. The skin of D6-deficient C57BL/6/129 (B6/129) mice, repeatedly treated with irritant, develops an exaggerated but self-limiting TNF- $\alpha$-dependent inflammatory pathology driven by D6-binding chemokines (29). The psoriasis-like appearance of the skin is characterized by unusually large numbers of epidermal $\mathrm{CD}^{+} \mathrm{T}$ cells and dermal mast cells (MCs) and is suppressed by $\mathrm{T}$ cell depletion (29).

The indispensable regulatory role played by D6 in the cutaneous iCC chemokine network led us to propose that, by suppressing inflammation, D6 may modulate susceptibility to de novo tumor formation and/or progression in the skin. The irritant employed in our previous study (29), 12-O-tetradecanoyl phorbol-13-acetate (TPA), is used as a tumor-promoting agent to drive de novo tumor emergence from mutagenized skin in mouse models of cutaneous malignancy, a response critically dependent on TNF- $\alpha$ (18) and pro- 
foundly influenced by $\mathrm{T}$ cells $(11,12)$. Using this model, we show that D6-deficient mice on 2 separate backgrounds (B6/129 and $\mathrm{FVB} / \mathrm{N}$ ) have increased susceptibility to de novo cutaneous tumor formation compared with WT counterparts. Strikingly, on a normally resistant background, deletion of $D 6$ is sufficient to allow the development of benign papillomas and the subsequent emergence of invasive squamous cell carcinoma (SCC). Moreover, transgenic expression of D6 in the basal epidermal layer of a susceptible mouse strain (K14-D6 mice; FVB/N background) increases keratinocyte sequestration of $\mathrm{iCC}$ chemokine and offers considerable protection from papilloma formation. Across all strains, tumor susceptibility directly correlated with the extent of $\mathrm{T}$ cell and $\mathrm{MC}$ recruitment after chemical application, 2 cell types with known protumorigenic activity in models of cutaneous malignancy. Thus, our data reveal the protumorigenic properties of the iCC chemokine network and identify D6-mediated chemokine sequestration as a mechanism of tumor suppression that we believe to be novel.

\section{Results}

D6 deficiency in B6/129 mice is sufficient to confer susceptibility to skin papillomas and invasive SCCs. Three consecutive daily applications of TPA (a single TPA application is ineffective) to dorsal skin of D6-deficient B6/129 mice induces exaggerated cutaneous inflammation that resolves after approximately 10 days (29). Repeated induction of this psoriasis-like response (after mutagenesis with 7,12-dimethylbenz(a)anthracene [DMBA]) may alter susceptibility to papilloma formation. Thus, D6-deficient and WT mice were given a single cutaneous application of DMBA followed by twelve 2-week cycles of TPA treatment, applying TPA on day 1, day 2, and day 3 of each cycle (protocol 1-2-3) (Figure 1A). In parallel, DMBAtreated mice received the same total dose of TPA but with 4- to 5-day rest periods between each treatment (protocol 1-5-10) (Figure 1B). This was done to assess the dependence of any phenotype on the psoriasis-like response. Most WT B6/129 animals, like B6 mice (32), remained papilloma free up to week 42 . In contrast, by week 21 of both regimens, papillomas had appeared on approximately $75 \%$ of D6-deficient mice (Figure 1 and Supplemental Figure 1A; supplemental material available online with this article; doi:10.1172/JCI30068DS1). After TPA discontinuation (week 25), some papillomas spontaneously resolved and others remained fairly constant in size, but many enlarged to form established papillomas greater than $5 \mathrm{~mm}$ in diameter $(55 \%-70 \%$ of D6-deficient mice by week 42, with 2-2.5 tumors per mouse on average; Figure 1, A and B). No WT animals developed these larger papillomas. In both regimens, differences in mean tumor burden between WT and D6-deficient animals became significant by week 19 for all papillomas $(P<0.01)$ and by week 24 for papillomas larger than $5 \mathrm{~mm}(P<0.05)$ and remained significantly different throughout the rest of the study. D6-deficient mice receiving carrier (acetone) alone in place of the DMBA or TPA failed to develop papillomas (data not shown). At week 42, large, flattened SCCs (diameter $\geq 8$ $\mathrm{mm}$ ), which were absent from WT mice, had developed from existing papillomas on 5 of 15 (protocol 1-2-3) and 4 of 15 (protocol 1-5-10) D6-deficient mice ( $P=0.021$ and $P=0.0498$, respectively [Fisher's exact test]). In all cases, tumor cells had invaded the dermis and were often present under the subcutaneous muscle layer (Supplemental Figure 1B). Two SCC-bearing D6-deficient animals, 1 from each treatment group, had keratinocyte deposits in draining inguinal lymph nodes, which in 1 case led to considerable disruption of normal nodal architecture (Supplemental Figure
1C). Thus, deletion of $D 6$ predisposed otherwise resistant $B 6 / 129$ mice to developing DMBA/TPA-induced skin tumors that had the potential to progress to invasive SCCs, able to metastasize to draining lymph nodes. This was independent of the acute psoriasis-like response that we previously observed in D6-deficient mice (29).

Further analysis of tumors at week 42 revealed that 17 of 19 papillomas (89\%) carried the H-ras codon 61 mutations (gln to leu) commonly found in DMBA/TPA-induced tumors (ref. 33; data not shown). Polymorphonucleocytes (PMNs) and $\mathrm{CD}^{+}$ $\mathrm{T}$ cells were present at high levels in most papillomas around the dermal/epidermal junction and within the hyperplastic epidermis (Supplemental Figure 1D and data not shown). This contrasted with uninvolved skin, which contained few PMNs or $\mathrm{CD}^{+}$cells. MCs and macrophages were found underlying tumors but at a frequency similar to that of uninvolved skin (data not shown). Similar infiltrates were found throughout SCCs: PMNs were present (data not shown), sometimes in large numbers, and all SCCs contained abundant $\mathrm{CD}^{+} \mathrm{T}$ cell populations principally within the epidermis (Supplemental Figure 1, E and F). Mice had not been exposed to TPA for approximately 17 weeks at the time of analysis, suggesting that tumors developed mechanisms of PMN and T cell recruitment independent of TPA.

Increased tumor susceptibility correlates with prolonged TPA-induced inflammation. The increased tumor susceptibility of D6-deficient mice was not dependent on the acute psoriasis-like response we previously reported (29). However, iCC chemokine clearance is delayed in D6-deficient mice even after a single TPA application (29), and this may subtly alter resolution of inflammation. Repeated induction of such a response (in protocol 1-5-10) could lead to progressive worsening of cutaneous inflammation in D6-deficient mice, and this may underlie the increased tumor susceptibility. Thus, D6-deficient and WT mice were treated with TPA according to the 1-5-10 protocol for 2 weeks and their skin examined on days 2 and 4 after the final TPA application alongside untreated controls. The psoriasis-like response was not evident (29), but D6-deficient mice exhibited prolonged inflammation and enhanced keratinocyte proliferation after the final TPA application (Figure 1, C-F), a response that was more marked than that observed after a single TPA application. This manifested as extended duration of skin thickening (Figure 1C), greater abundance of $\mathrm{Ki}^{+} 7^{+}$keratinocytes in basal and suprabasal layers (Figure 1D), and more epidermal $\mathrm{CD}^{+} \mathrm{T}$ cells (Figure 1E), and dermal MCs (Figure $1 F)$. PMN and macrophage numbers were not significantly different between WT and D6-deficient mice (data not shown). Carrier-treated $D 6$-deficient and WT mice had few cutaneous MCs, epidermal CD3+ T cells, or Ki67+ keratinocytes. Next, WT and D6deficient mice were treated with TPA (days 1, 5, and 10) and eluates prepared on day 12 from dorsal skin biopsies to compare bioavailable chemokine levels (29). Of 6 chemokines assayed (CCL2, -3, and -5 and CXC chemokine ligands [CXCLs] 1, 9, and 10), only CCL3 was consistently detectable and was dramatically elevated in D6-deficient mice compared with WT mice (Figure 1G). No chemokines were detected in skin eluates from untreated mice (data not shown). Thus, susceptibility to papillomas was linked to raised CCL3 levels, perturbed inflammation, and enhanced keratinocyte proliferation in D6-deficient mice.

D6 deficiency enhances papilloma formation in susceptible $\mathrm{FVB} / \mathrm{N}$ mice. To examine whether D6 modifies inflammation and papilloma susceptibility in mice already predisposed to these tumors, D6-deficient mice were backcrossed for 6 generations with FVB/N 

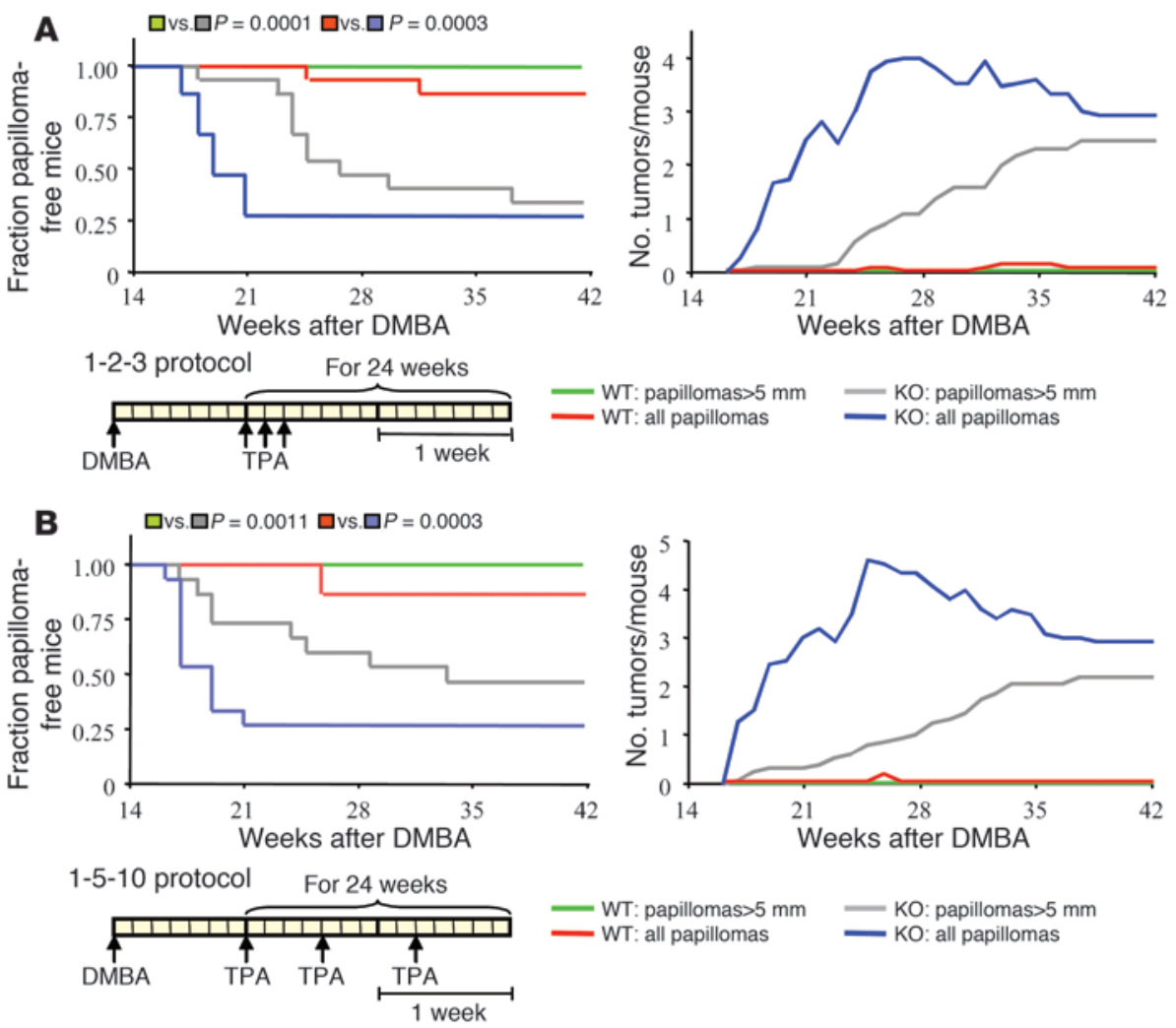

C

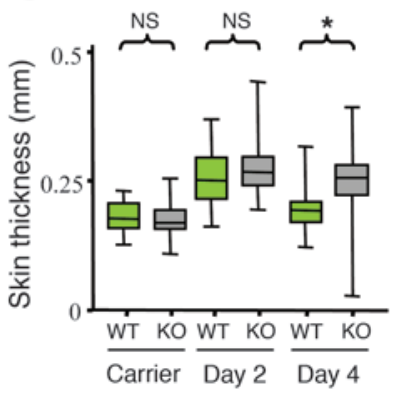

D
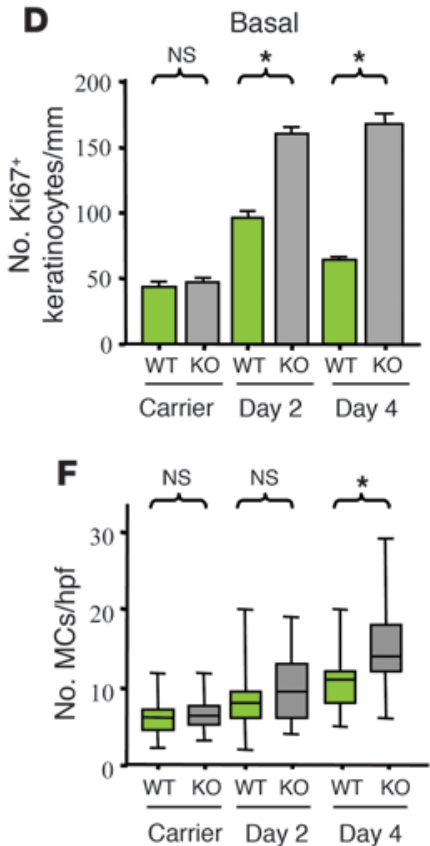

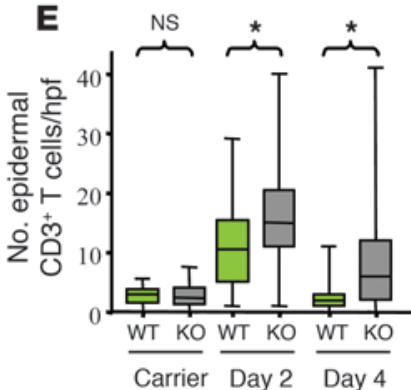

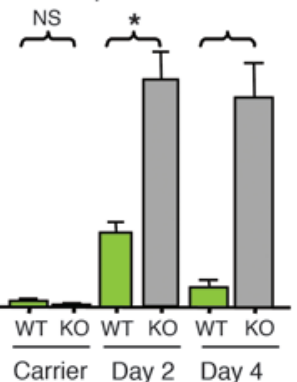

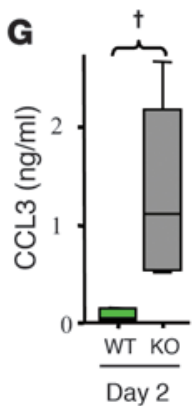

\section{Figure 1}

$D 6$ deficiency confers susceptibility to skin tumors in B6/129 mice. (A and B) Fifteen DMBA-treated D6-deficient (KO) or WT mice received TPA for 24 weeks according to protocols shown. Total papillomas and papillomas greater than $5 \mathrm{~mm}$ in diameter were counted once a week. Fraction of papilloma-free mice (left) and mean tumor burden per mouse (right) are shown. Log-rank tests were applied to data in left panel. See Results for results of statistical tests on tumor burden data. (C-F) D6-deficient and WT mice were treated with TPA on days 1, 5, and 10, and skin was harvested 2 or 4 days later and scored for skin thickness (dermis plus epidermis) (C), Ki67+ keratinocytes (basal and suprabasal layers) (D), epidermal CD3 ${ }^{+} \mathrm{T}$ cells $(E)$, and MCs (F). Dorsal skin of "carrier" control mice was harvested 2 days after application of carrier (acetone) and similarly assessed. (C, E, and F) A total of 25-40 data points collected from stained sections from 5 TPA-treated mice or 4-5 carrier controls of each genotype per time point. (D) The number of $\mathrm{Ki}^{+} 7^{+}$cells per millimeter was determined by counting 5 randomly selected $400-\mu \mathrm{m}$ regions of epidermis from stained sections from 5 TPA-treated mice or 4-5 carrier controls of each genotype per time point. Data are representative of 3 repeated experiments and were analyzed using a 2-tailed unpaired Student's $t$ test. (G) Seven D6-deficient and WT mice were treated with TPA on days 1,5 , and 10, skin was harvested 2 days later, chemokine concentrations in eluates were determined using Luminex technology, and data were analyzed using a 2-tailed unpaired Student's $t$ test. A repeat experiment generated a similar result. hpf, high-powered field. ${ }^{*} P<0.001 ;{ }^{\dagger} P<0.01$. mice, a strain particularly prone to DMBA/TPA-induced tumors (32). Strikingly, protocol 1-2-3 failed to induce the psoriasis-like response in D6-deficient mice on this background. While there was no significant difference in skin thickening or leukocyte infiltration between WT and D6-deficient skin harvested on day 2 after the last TPA application, there were more $\mathrm{Ki} 67^{+}$keratinocytes present in the basal layer at this time point (Figure 2, A-D, and data not shown). Moreover, compared with WT skin, skin from $D 6$-defi- cient FVB mice was more thickened on day 4 after TPA, had more proliferating basal keratinocytes, and contained increased numbers of epidermal CD3 ${ }^{+} \mathrm{T}$ cells and dermal MCs. Thus, though less marked than in B6/129 mice, deletion of D6 extends inflammation and enhances keratinocyte proliferation in FVB mice.

To examine tumor susceptibility, DMBA-treated D6-deficient and WT FVB/N mice were treated twice a week with TPA for 20 weeks, and papillomas were scored (Figure 2E). The proportion of 
A
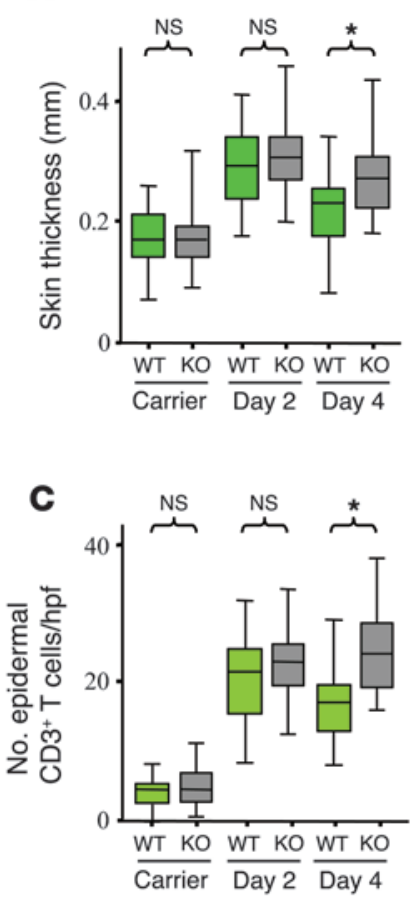
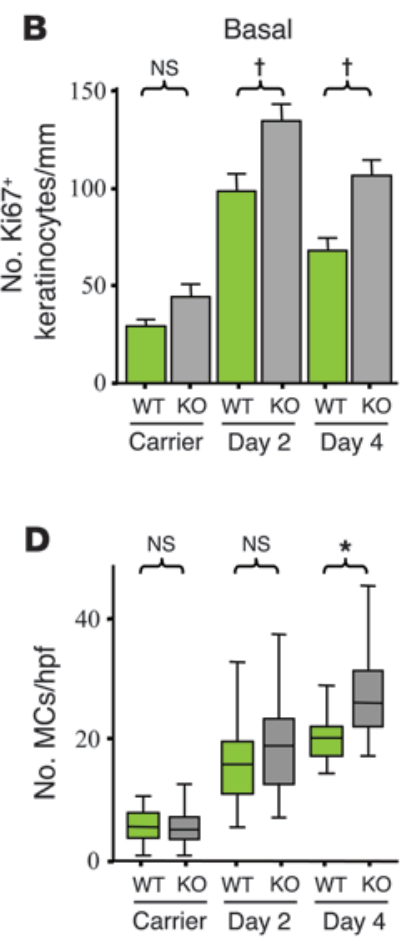
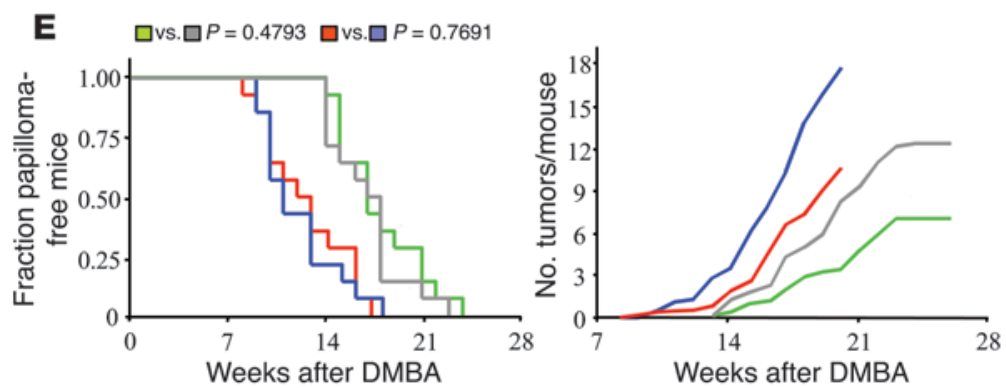

$2 \times /$ week protocol
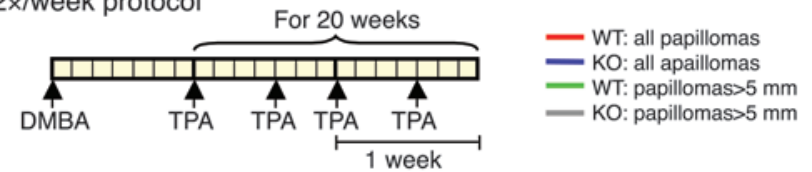

28

(1)

\section{hu} man SCC formation, D6-expressing cells would be expected to be associated with SCCs and their precursors. Thus, we performed immunohistochemistry on 9 SCCs and 3 dysplasias from the oral cavity. Oral SCC formation bears similarities to the DMBA/TPA model because while human skin tumors usually develop as a result of UV exposure, those in the oral cavity pri-

3 times a week to give more regular inflammatory stimulation to DMBA-treated skin (Supplemental Figure 2). Again, D6-deficient mice had more papillomas than WT mice, though this was less marked than when TPA was only applied twice a week. Together, these data support observations made in B6/129 mice that $D 6$ suppresses $\mathrm{MC}$ and $\mathrm{CD}^{+} \mathrm{T}$ cell abundance in the skin, limits keratinocyte proliferation, and suppresses tumor formation.

D6 expression by LECs associated with SCCs in humans. If D6 has the potential to play an equivalent role in

WT and D6-deficient mice that remained tumor-free decreased at a similar rate, yet $D 6$-deficient mice developed more tumors than their WT counterparts. Differences between WT and D6-deficient animals became significant on week 15 for all papillomas $(P<0.05)$ and week 18 when tumors greater than $5 \mathrm{~mm}$ in diameter were scored $(P<0.05)$. These differences remained significant throughout the experiment. On WT and D6-deficient mice, a similar proportion of smaller tumors ( $40 \%$ ) formed papillomas of greater than $5 \mathrm{~mm}$ in diameter, and there was no marked difference in average tumor size. Analysis of D6-deficient mice at week 27 revealed that, as in WT mice, most papillomas (11/12; 92\%) harbored $\mathrm{H}$-ras codon 61 mutations and contained considerable infiltration of PMNs and $\mathrm{CD}^{+} \mathrm{T}$ cells (data not shown). Ten WT and $D 6$-deficient animals were kept for a further 15 weeks. Invasive tumors developed on 4 WT and 5 D6-deficient mice, and log-rank analysis failed to reveal any significant change in rate of conversion to SCC (data not shown). In parallel experiments, TPA was applied marily arise due to repeated exposure to mutagenic and inflammatory chemicals, particularly those found in tobacco smoke (34). Moreover, chronic oral inflammation predisposes to SCC (35), and extensive leukocyte infiltrates are associated with oral SCCs and dysplasias. In comparison with isotype controls, specific D6 immunoreactivity was not observed on tumor or epithelial cells, but in all 12 samples, as has been found in normal human skin and other tissues (20), it was readily detectable on LECs that lined a subset of lymphatic channels identifiable by their often flattened appearance, lack of red blood cells, and absence of obvious mural or smooth muscle cells (Supplemental Figure 3). As previously shown (20), occasional isolated dermal cells that were likely leukocytes also showed D6 immunoreactivity. Weakly stained D6 ${ }^{+}$LECs were found throughout the dermis in all samples (Supplemental Figure $3 \mathrm{~A})$, but notably, in all SCCs, there was a marked increase in the intensity of D6 LEC immunostaining around invasive tumor cells (Supplemental Figure 3, B-G), with LECs sometimes appearing 


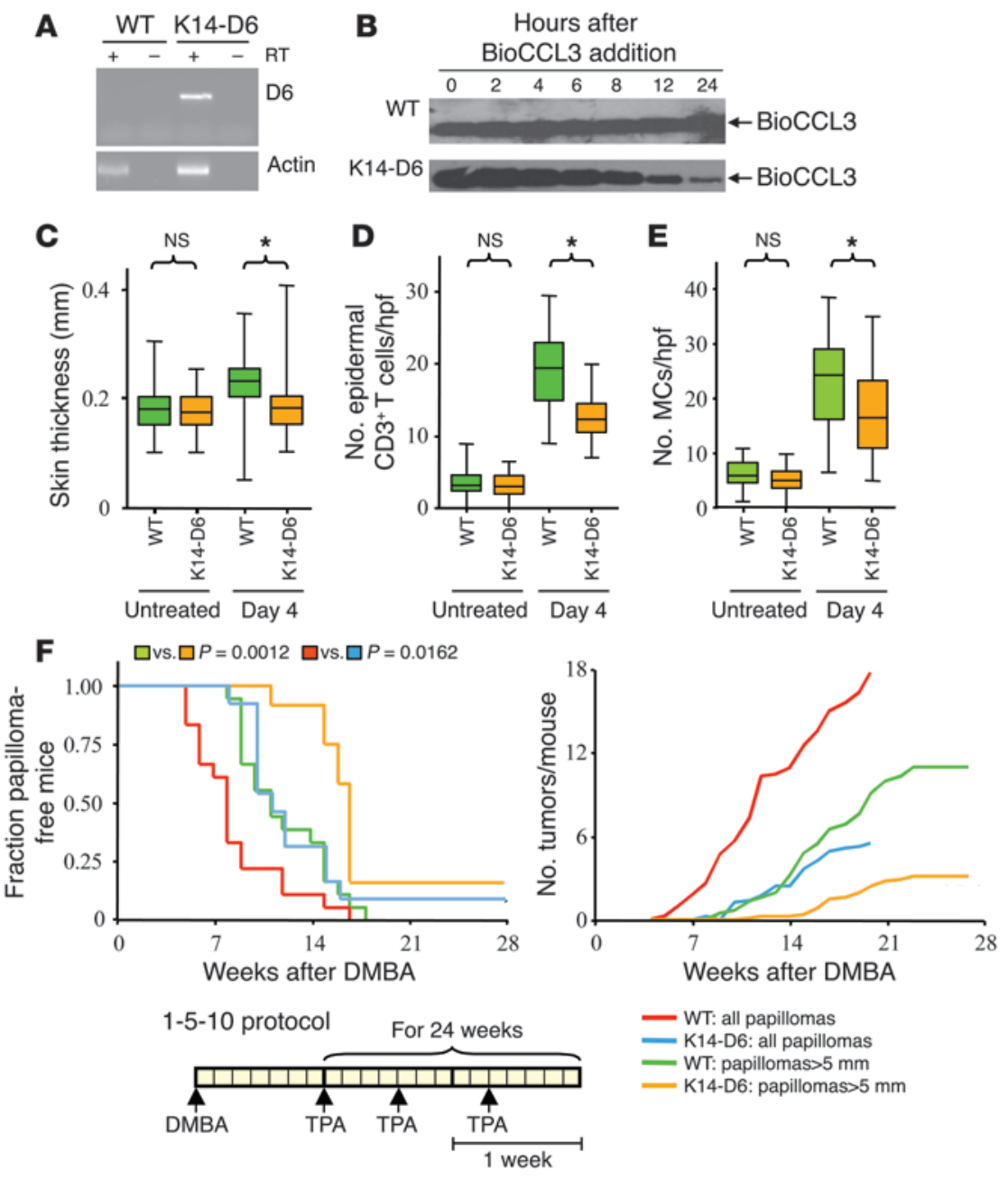

\section{Figure 3}

Transgenic expression of D6 in the epidermis of FVB/N mice dampens cutaneous inflammation and suppresses papilloma formation. (A) RNA from epidermal sheets from dorsal skin of WT and K14-D6 mice was subjected to RT-PCR (with or without reverse transcriptase $[R T])$ for mouse D6 or actin. Products were electrophoresed on an agarose gel and visualized with ethidium bromide under UV light. (B) Keratinocytes $\left(2.5 \times 10^{5}\right)$ from neonatal K14D6 or WT mice were cultured in $1 \mathrm{ml}$ of medium containing $10 \mathrm{nM} \mathrm{BioCCL3}$, and remaining BioCCL3 was detected by western blotting at the times indicated. Data are representative of 3 repeated experiments. (C-E) WT and K14D6 mice ( $n=5$ per group) received TPA on 3 consecutive days, and dorsal skin was harvested 4 days later. Untreated mice of each genotype ( $n=5 /$ group) were shaved and dorsal skin immediately harvested. Processed sections were scored for skin thickness (dermis plus epidermis) (C), epidermal $\mathrm{CD}^{+} \mathrm{T}$ cells (D), and MCs (E). A total of 25-40 data points were collected for each parameter from sections from 5 mice of each genotype. Data were analyzed using a 2-tailed unpaired Student's $t$ test. A repeat experiment generated similar data. (F) Fifteen K14-D6 mice and 17 WT counterparts received DMBA, and then TPA on days 1,5 , and 10 every 2 weeks for 20 weeks. Papillomas greater than $5 \mathrm{~mm}$ in diameter were counted once a week. The fraction of papilloma-free mice (left) and the mean tumor burden per mouse (right) are shown. Log-rank tests were applied to the data in the left panel. See Results for results of statistical tests on tumor burden data. ${ }^{*} P<0.001$. to be in direct physical contact with the invading tumor cells (e.g., Supplemental Figure 3, B, D, and G). While these observations do not directly demonstrate a role for D6 in controlling human SCC development, they do place D6 in the correct anatomical location to limit inflammation during SCC formation.

Transgenic expression of D 6 in keratinocytes dampens TPA-induced inflammation and decreases susceptibility of $F V B / N$ mice to skin tumors. We anticipated that ectopic D6 expression would suppress cutaneous inflammation and reduce papilloma formation, so we generated FVB/N K14-D6 mice carrying a murine D6 transgene under the control of the human keratin 14 (K14) promoter. This promoter directs transgene expression to the basal keratinocyte layer, and epidermal D6 expression in K14-D6 mice was confirmed by RT-PCR (Figure 3A). To directly assess the activity of the product of the D6 transgene, we assessed the ability of cultured keratinocytes to remove extracellular biotinylated CCL3 (BioCCL3). Consistent with chemokine sequestration by D6, K14-D6 keratinocytes directed progressive depletion of BioCCL3, while equivalent WT cultures left it intact (Figure 3B). A 10-fold molar excess of unlabeled CCL22, a high-affinity D6 ligand (22), prevented BioCCL3 depletion by K14-D6 keratinocytes (data not shown). Next, we examined the response of K14-D6 and WT mice to cutaneous TPA application (protocol 1-2-3). On day 4 after the last application, the skin of WT mice was still markedly thick- ened, while in K14-D6 mice it had returned to normal resting thickness (Figure 3C). In addition, despite the presence of similar leukocyte infiltrates early after TPA application (not shown), by day 4 following TPA treatment, K14-D6 mouse skin contained fewer epidermal $\mathrm{CD}^{+}$cells and dermal MCs than did WT skin (Figure 3, D and E). Thus, K14-D6 mice carry keratinocytes capable of inflammatory CC chemokine depletion and more rapidly resolve TPA-induced inflammation than WT mice.

Next, DMBA-treated K14-D6 and WT mice received TPA for 20 weeks according to protocol 1-5-10. Under these conditions, K14D6 mice were protected against papillomas (Figure 3F). Compared with WT mice, tumor emergence was delayed by approximately 6 weeks in K14-D6 mice, and tumor burden reduced by approximately $70 \%$, achieving statistical significance $(P<0.05)$ at weeks 8 (all papillomas) and 12 (papillomas larger than $5 \mathrm{~mm}$ in diameter). Notably, 20\% (3/15) of K14-D6 mice failed to develop any papillomas greater than $5 \mathrm{~mm}$ in diameter up to 27 weeks after DMBA initiation, and even small papillomas failed to develop on 2 of these animals. On week $27,33 \%(5 / 15)$ of the WT animals but only $7 \%(1 / 15)$ of the K14-D6 group carried SCCs that had detectably invaded into the dermis and/or through the subcutaneous muscle layer $(P=0.084$; Fisher's exact test). Unlike uninvolved skin, papillomas and SCCs from mice of both genotypes contained abundant infiltrates of PMNs and $\mathrm{CD}^{+} \mathrm{T}$ cells (data not shown). Thus, sup- 

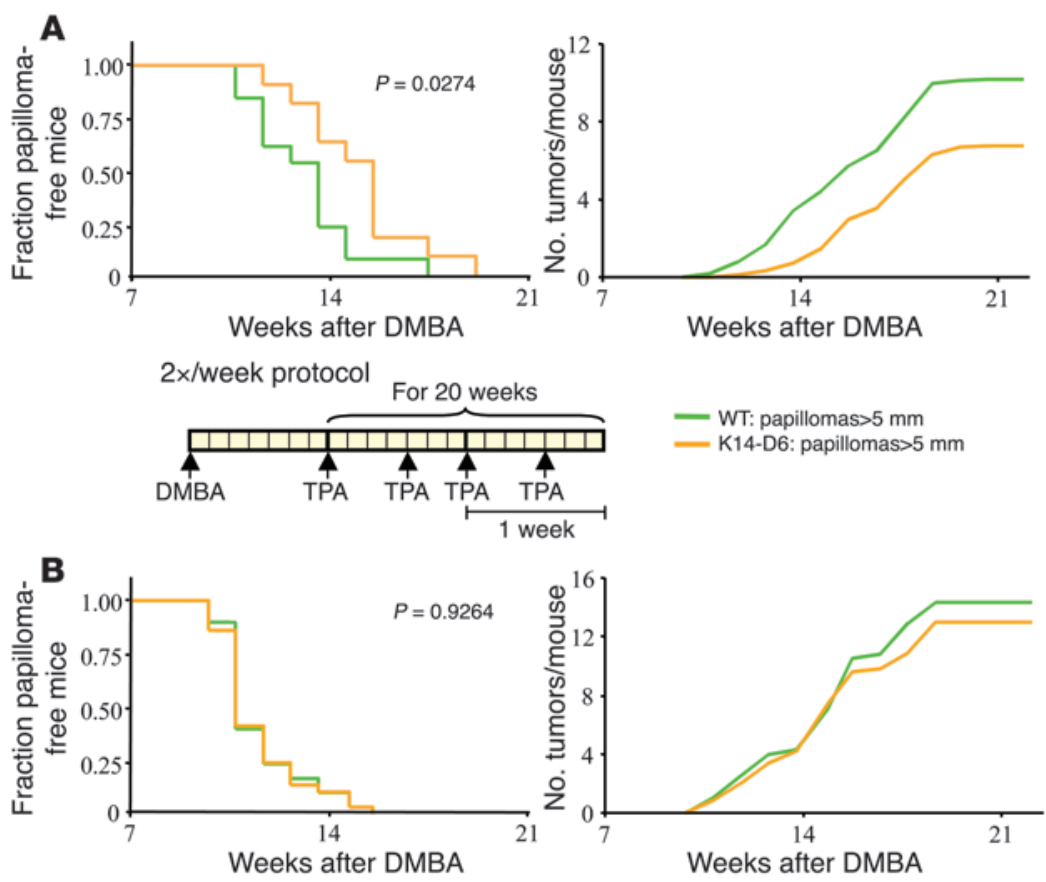

$3 \times /$ week protocol
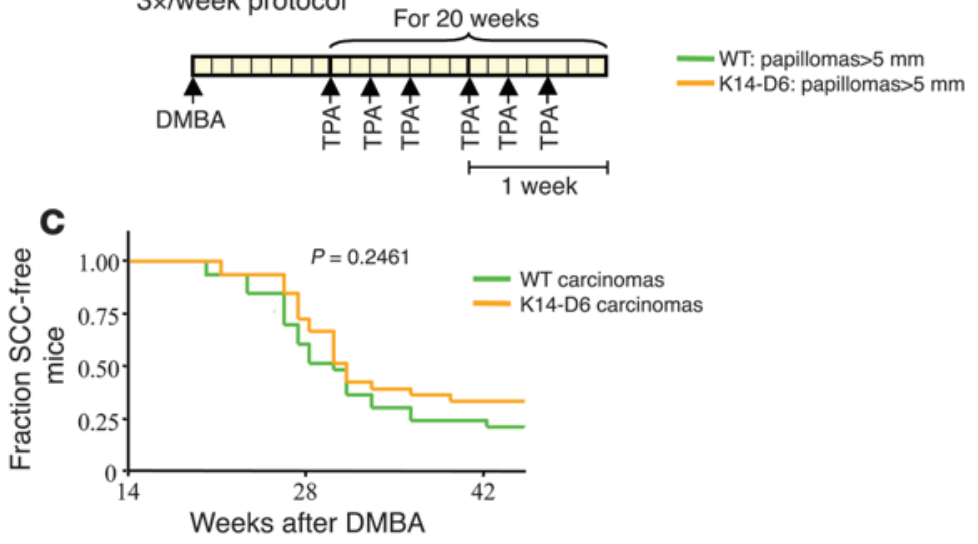

pression of early tumor formation can be augmented by increased D6 expression (in this case, using an ectopic D6 transgene), indicating that iCC chemokines contribute to skin tumor development in WT animals even in the presence of endogenous D6.

Relative protection from SCC development in K14-D6 mice may be due to low papilloma burden rather than a direct effect on tumor progression. Since differences in responses to TPA between WT and K14-D6 were most apparent 3-4 days after TPA application, more regular TPA application may override protection by the D6 transgene by removing the temporal window in which it operates. Thus, the frequency of application was increased to 2 or 3 times per week. K14-D6 mice in the twice-per-week protocol still showed some protection relative to WT (Figure 4A). On average, papillomas greater than $5 \mathrm{~mm}$ in diameter emerged a few weeks later on K14-D6 mice, and there was an approximately 30\% reduction in mean tumor burden that became statistically significant by week $14(P<0.05)$ and remained significantly different throughout the experiment. However, the transgene was not protective when TPA was applied 3 times per week (Figure 4B). After completion of the TPA treatments, these animals were observed for a further 20

\section{Figure 4}

The K14-D6 transgene can be overwhelmed by increasing the frequency of TPA application and does not affect conversion of papillomas to SCC. DMBA-treated K14-D6 or WT mice received TPA for 20 weeks either 2 times per week (A; 13 mice per genotype) or 3 times per week (B and $\mathbf{C}$; 29 mice per genotype). (A and B) Papillomas greater than $5 \mathrm{~mm}$ in diameter were counted once a week. The fraction of papilloma-free mice (left) and mean tumor burden per mouse (right) are shown. (C) Mice used to generate data shown in B were followed for 45 weeks and assessed every week for SCC formation. Tumors that had the morphological appearance of SCCs were processed for histology and scored as SCCs when invasion into the dermis was detectable. Log-rank tests were applied to data in the left panels. See Results for results of statistical tests on tumor burden data.

weeks. Papillomas that developed the morphological appearance of carcinomas were harvested and classified as SCCs upon confirmation of dermal invasion. Under these conditions, there was no significant difference in the rate of SCC emergence between WT and K14-D6 mice (Figure 4C). Thus, protection by the D6 transgene was overwhelmed by increased frequency of inflammatory stimulation, and the D6 transgene did not influence rate of conversion to SCC.

\section{Discussion}

Proinflammatory CC chemokines control inflammatory leukocyte recruitment and behavior by signaling through chemokine receptors CCR1 to -5 . Many of the ligands for these receptors also bind to D6. While signaling through D6 in some scenarios cannot be excluded, existing evidence strongly supports the notion that constitutive, ligandindependent cycling to and from the cell surface drives sequestration of $\mathrm{iCC}$ chemokines by this receptor. In the skin, D6 limits chemokine bioavailability and is critical for resolution of inflammation $(23,29,30)$. Here we show that (a) endogenous D6 is sufficiently active during resolution to enable it to mediate significant suppression of tumor formation in the skin; and (b) D6 transgenesis can be used to effectively limit tumorigenesis. Our data highlight the critical importance of the iCC chemokine network in the early stages of a progressive, multistep mouse model of de novo tumorigenesis that has many similarities to human cancer development.

In D6-deficient mice on 2 distinct genetic backgrounds, tumor susceptibility correlated with the prolonged presence of epidermal $\mathrm{CD}^{+} \mathrm{T}$ cells and dermal MCs after TPA application. Conversely, in K14-D6 mice, these cells were markedly reduced in inflamed skin, coinciding with decreased tumor susceptibility. As D6 controls cutaneous iCC chemokine abundance (29), the simplest explanation for these observations is that D6 regulates the level of iCC chemokines to directly affect MC and T cell recruitment. Antigenexperienced $\mathrm{T}$ cell subsets often express one or more of the receptors CCR 1 to -5 , and CCR4 has been specifically implicated in cutaneous T cell homing $(36,37)$. Mouse MCs and their progeni- 
tors also likely fall under the migratory control of D6 ligands; this is certainly the case for human MCs (38). Significantly, MCs and $\mathrm{T}$ cells show protumorigenic activity in cutaneous malignancy, thus providing a likely cellular link between $D 6$ dose and tumor development. MCs can contribute to tumor-associated angiogenesis (8, 39), while TCR $\alpha \beta^{+} \mathrm{T}$ cells, the bulk of the epidermal CD3 ${ }^{+} \mathrm{T}$ cells we detected (29), enhance tumor burden in the DMBA/TPA model $(12,40)$ and in K14-HPV16 mice (which express human papilloma virus early region genes in basal keratinocytes and spontaneously develop SCCs) (5). Interestingly, MC degranulation is dependent on protein kinase $\mathrm{C}$ isoforms (direct targets of TPA; ref. 41), and phorbol esters are potent $\mathrm{T}$ cell activators. Thus, the number of $\mathrm{T}$ cells and MCs in the skin during each TPA application may determine the dose of putative protumorigenic leukocyte-derived factors that are released in the skin. Chemokines may also directly participate in leukocyte activation. CCL3, a D6 ligand with defective clearance kinetics in D6-deficient skin (ref. 29 and Figure 1G), can directly contribute to MC degranulation through CCR1 (41, 42). However, while molecular insights are emerging into how leukocytes encourage tumorigenesis (9), the identity of protumorigenic factors released by these cells remains a key objective.

Leukocytes are abundant in established DMBA/TPA-induced tumors, and some leukocyte subsets, such as TCR $\alpha \beta^{+} \mathrm{T}$ cells, enhance progression to carcinoma (12). The near absence of papillomas on WT B6/129 mice means we cannot exclude that D6 affects transition to carcinomas in this strain, but our data with FVB/N mice suggest that endogenous and transgenic D6 principally influence early tumor formation. This may be due to established tumors evolving TPA-independent mechanisms of leukocyte recruitment that override the protective effects of D6. If non-D6binding chemoattractants mediate this effect, D6 will evidently be unable to control their bioavailability. For example, proinflammatory CXC chemokines may be involved - PMNs, abundant in DMBA/TPA-induced tumors, can be recruited via CXCR2, and CXCR2 ligand expression can be driven by activated ras found in these tumors (43). CXCR2 also controls angiogenesis (44), and interestingly, DARC, another atypical chemokine receptor functionally related to D6, suppresses the angiogenic activity of CXCR2 ligands to limit spontaneous prostate tumor growth in mice (17). Alternatively, established DMBA/TPA-induced tumors may switch on continuous iCC chemokine production. Even when transgenically overexpressed, D6 may not be able to neutralize sufficient quantities of these chemokines to have an impact on recruitment and/or activation of protumorigenic leukocytes. In this regard, it is notable that increased frequency of TPA application (which will increase the regularity of chemokine induction) can override protection by the D6 transgene and reduce differences in tumor burden between D6-deficient and WT FVB/N mice (Figure 4 and Supplemental Figure 2). In such protocols, the resolution phase of the inflammatory response becomes less relevant to the eventual outcome, and it is in this phase that D6 is functionally significant. Likewise, established papillomas constitutively producing iCC chemokines will not provide a resolution phase for D6 to exert its antiinflammatory function.

In conclusion, our data reveal the importance of D6 as a regulator of early tumor formation in mice. It will now be important to determine the nature of the involvement of D6 in human tumorigenesis, which is likely to be of particular relevance to those tumors in which inflammation has been directly implicated in the tumorigenic process. Moreover, while D6 does not influence progression to carcinoma in the DMBA/TPA model, its ability to influence leukocyte infiltration may affect tumor progression in other mouse tumor models or in emerging human tumors. D6 ${ }^{+}$LECs are found around SCCs (Supplemental Figure 3) and other solid human tumors (e.g., endometrial carcinomas; R.J.B. Nibbs, unpublished observations), while in some angiosarcomas (20) and most Kaposi sarcomas (R.J.B. Nibbs, unpublished observations), D6 is highly expressed by the tumor cells themselves, most likely due to their derivation from LECs (45). It is tempting to speculate that chemokine sequestration by D6 in these tumors may determine the precise nature of the inflammatory infiltrate, be it pro- or antitumorigenic. Detailed analyses of human cancers and other mouse models are required to address these issues. However, our current data provide further rationale for exploring the therapeutic potential of iCC chemokine inhibition in human tumorigenesis.

\section{Methods}

Animals. Experiments were performed on age-matched female littermates older than 8 weeks of age at the start of the experiment. D6-deficient B6/129 animals were generated by Donald Cook (National Institute of Environmental Health Sciences, Research Triangle Park, North Carolina, USA) and Sergio Lira (Mount Sinai School of Medicine, New York, New York, USA) and maintained along with WT counterparts as previously described (29). Mice were backcrossed for 6 generations with FVB/N mice (Harlan) and heterozygotes crossbred to generate D6-deficient and WT offspring. To generate K14-D6 mice, the pG32.K14 vector (E. Fuchs, Rockefeller University, New York, New York, USA) was modified such that the murine D6 open reading frame was preceded by the human K14 promoter and rabbit $\beta$-globin intron and followed by the human K14 3' untranslated region and poly(A) signal (46). This was introduced into $\mathrm{d} 0.5 \mathrm{FVB} / \mathrm{N}$ embryos by pronuclear injection, and surviving embryos were transferred into oviducts of pseudopregnant foster mice. Transgenic offspring were identified by PCR from biopsy DNA (primers CTCTTCACTGATCTCCCTCCAC and ATGACAAGCTCCAAAGAGATGC). Female offspring of 2 independent founders were assessed for susceptibility to papilloma formation (TPA protocol 1-5-10). Transgene-positive offspring from both founders were protected from papillomas relative to WT littermates (Figure 3E and data not shown). All subsequent experiments were performed using females derived from the founder whose offspring were used to generate the data shown in Figure 3E. Mice were kept in specific pathogen-free conditions under the authority of United Kingdom government Home Office licenses. Protocols were approved by Glasgow University Ethical Review Panel.

RT-PCR analysis of epidermal sheets. Dorsal skin was dissected, fat removed, and the skin sliced thinly and placed dermal side down in $25 \mathrm{~mm}$ EDTA in PBS for 2 hours at $37^{\circ} \mathrm{C}$ to release epidermal sheets. RNA from samples with clear histological evidence of epidermal separation were treated with DNaseI and used in RT-PCR. First-strand synthesis was done with a SuperScript kit (Invitrogen) with or without reverse transcriptase. PCR was done using primers for D6 (AGCTTTACCTGCTGAACCTGG and AAGAAGATCATGGCCAAGAGTG) and actin (TGAACCCTAAGGCCAACCGTG and GCTCATAGCTCTTCTCCAGGG) using Prealiquoted ReddyMix PCR Master Mix (ABgene) and 30 cycles of $94^{\circ} \mathrm{C}(15 \mathrm{~s}), 55^{\circ} \mathrm{C}(15 \mathrm{~s}), 72^{\circ} \mathrm{C}$ (30 s). Products were electrophoresed on a $1 \%$ agarose gel containing ethidium bromide and visualized under UV light.

Keratinocyte culture and BioCCL3 depletion. Keratinocyte cultures were prepared as described in ref. 47 from individual neonates from a K14-D6 $\times$ WT cross. After genotyping tail biopsies, $2.5 \times 10^{5}$ keratinocytes were cultured in $1 \mathrm{ml}$ Keratinocyte Growth Medium (Cambrex) with 1\% bovine serum albumin and $10 \mathrm{nM}$ BioCCL3 (28). Fifteen microliters of heat-denatured aliquots of culture medium were electrophoresed and western blots pre- 
pared (28). BioCCL3 was detected using streptavidin-horseradish peroxidase conjugate (Dako) and visualized with SuperSignal West Femto Maximum Sensitivity Substrate (Pierce Biotechnology).

Induction of cutaneous inflammation and tumors. Skin tumors were induced as previously described (48). Dorsal skin was shaved and the next day 25 $\mu \mathrm{g}$ DMBA (Sigma-Aldrich) applied in $200 \mu \mathrm{l}$ of acetone. A week later, TPA applications were initiated and continued for 20-24 weeks, each time applying $7.6 \mathrm{nmol}$ TPA (Sigma-Aldrich) in $150 \mu \mathrm{l}$ acetone. Dorsal skin was kept hair free by regular shaving. Emergent papillomas were scored once a week. Short-term TPA treatments to assess cutaneous inflammation were done similarly but without the DMBA step, beginning TPA applications the day after shaving. Papillomas, SCCs, and inflamed skin were harvested, processed, and immunostained as previously described (29). Anti-Ki67 was from Novocastra. Slides were examined with an Axiostar Plus microscope (Zeiss), and images were captured using Axiovision 3 software (Zeiss). To determine skin thickness (dermis plus epidermis), at least 20 random $\times 20$ magnification fields from $\mathrm{H} \& \mathrm{E}$-stained sections from at least 5 mice per genotype were scored using an eyepiece graticule. Leukocyte counts were determined from immunostained sections of skin taken from at least 5 mice per genotype, scoring 25-40 random fields from 5 mice per genotype under $\times 20$ magnification. Ki67+ basal and suprabasal cells were scored in $5 \times 400 \mu \mathrm{m}$ high-powered fields per mouse, with 5 mice per group. For box-and-whisker plots, the box indicates a $95 \%$ confidence interval around the mean (line across the box), and the whiskers indicate the range of the furthest outlying points. For interstitial chemokine measurements, $10 \times 2$ $\mathrm{mm}$ punch biopsies from inflamed dorsal skin from each mouse were incubated for 2 hours in $400 \mu \mathrm{PBS}$ containing protease inhibitors at $4^{\circ} \mathrm{C}(29)$ and chemokine concentrations in the eluates determined using Luminex technology with a base kit and detection reagents for CCL2, -3 , and -5 and CXCL1, -9 , and -10 from BioSource (Invitrogen) according to the manufacturer's instructions. Tumor burden data for B6/129 mice were assessed at each time point using a Wilcoxon signed-rank test in comparison with a hypothetical mean of 0 . Other data were analyzed using GraphPad Prism 4.0 software (GraphPad Software Inc.) using the log-rank test (survival curves), Fisher's exact test (where indicated in Results), or 2-tailed Student's $t$ test (all other data). $P$ values less than 0.05 were considered significant.

Immunohistochemistry on human SCC. Sections of oral SCC and dysplasias, obtained by K.D. Hunter under existing ethical approval (with informed consent) granted by the Glasgow Dental Hospital Area Ethics Committee (49) were stained and counterstained with anti-D6 antibodies and hematoxylin, respectively (20). Multiple images were captured per section with settings unchanged between images to allow comparison of immunostaining intensity at different sites.

\section{Acknowledgments}

The authors thank Stephen Bell and his team (particularly Maria Hendry and Nicola McAllister), Sheila Bryson at the Beatson Transgenic Service, A. Gracie (for assistance with Luminex assays), and D. Greenhalgh (for advice on keratinocyte isolation). R.J.B. Nibbs thanks A.M. Wilson for excellent support services. This work was funded by Cancer Research UK. V. King is supported by the Oliver Bird Rheumatism Programme.

Received for publication August 15, 2006, and accepted in revised form April 10, 2007.

Address correspondence to: Robert Nibbs or Gerard Graham, Division of Immunology, Infection and Inflammation, Glasgow Biomedical Research Centre, 120 University Place, Glasgow University, Glasgow G12 8TA, United Kingdom. Phone: 44-141-330-3960 or 44-141-330-3982; Fax: 44-141-330-4297; E-mail: r.nibbs@clinmed. gla.ac.uk (R. Nibbs); g.graham@clinmed.gla.ac.uk (G. Graham).
1. Rossi, D., and Zlotnik, A. 2000. The biology of chemokines and their receptors. Annu. Rev. Immunol. 18:217-242.

2. de Visser, K.E., Eichten, A., and Coussens, L.M. 2006. Paradoxical roles of the immune system during cancer development. Nat. Rev. Cancer. 6:24-37.

3. Balkwill, F., Charles, K.A., and Mantovani, A. 2005. Smoldering and polarized inflammation in the initiation and promotion of malignant disease. Cancer Cell. 7:211-217.

4. Pikarsky, E., et al. 2004. NF-kappaB functions as a tumour promoter in inflammation-associated cancer. Nature. 431:461-466.

5. Daniel, D., et al. 2003. Immune enhancement of skin carcinogenesis by CD4+ T cells. J. Exp. Med. 197:1017-1028.

6. Greten, F.R., et al. 2004. IKKbeta links inflammation and tumorigenesis in a mouse model of colitis-associated cancer. Cell. 118:285-296.

7. Houghton, J., et al. 2004. Gastric cancer originating from bone marrow-derived cells. Science. 306:1568-1571.

8. Coussens, L.M., et al. 1999. Inflammatory mast cells up-regulate angiogenesis during squamous epithelial carcinogenesis. Genes Dev. 13:1382-1397.

9. Coussens, L.M., Tinkle, C.L., Hanahan, D., and Werb, Z. 2000. MMP-9 supplied by bone marrowderived cells contributes to skin carcinogenesis. Cell. 103:481-490.

10. de Visser, K.E., Korets, L.V., and Coussens, L.M. 2005. De novo carcinogenesis promoted by chronic inflammation is B lymphocyte dependent. Cancer Cell. 7:411-423.

11. Girardi, M., et al. 2001. Regulation of cutaneous malignancy by gammadelta $\mathrm{T}$ cells. Science. 294:605-609.

12. Girardi, M., et al. 2003. The distinct contributions of murine $\mathrm{T}$ cell receptor (TCR)gammadelta ${ }^{+}$and TCRalphabeta $+\mathrm{T}$ cells to different stages of chemically induced skin cancer. J. Exp. Med. 198:747-755.

13. Lin, E.Y., Nguyen, A.V., Russell, R.G., and Pollard, J.W. 2001. Colony-stimulating factor 1 promotes progression of mammary tumors to malignancy. J. Exp. Med. 193:727-740.

14. Balkwill, F. 2004. Cancer and the chemokine network. Nat. Rev. Cancer. 4:540-550.

15. Conti, I., and Rollins, B.J. 2004. CCL2 (monocyte chemoattractant protein-1) and cancer. Semin. Cancer Biol. 14:149-154.

16. Mantovani, A., et al. 2004. Chemokines in the recruitment and shaping of the leukocyte infiltrate of tumors. Semin. Cancer Biol. 14:155-160.

17. Shen, H., Schuster, R., Stringer, K.F., Waltz, S.E., and Lentsch, A.B. 2006. The Duffy antigen/receptor for chemokines (DARC) regulates prostate tumor growth. FASEB J. 20:59-64.

18. Moore, R.J., et al. 1999. Mice deficient in tumor necrosis factor-alpha are resistant to skin carcinogenesis. Nat. Med. 5:828-831.

19. Yang, X., et al. 2006. Essential contribution of a chemokine, CCL3, and its receptor, CCR1, to hepatocellular carcinoma progression. Int. J. Cancer. 118:1869-1876.

20. Nibbs, R.J., et al. 2001. The beta-chemokine receptor D6 is expressed by lymphatic endothelium and a subset of vascular tumors. Am. J. Pathol. 158:867-877.

21. Nibbs, R.J., Wylie, S.M., Yang, J., Landau, N.R., and Graham, G.J. 1997. Cloning and characterization of a novel promiscuous human beta-chemokine receptor D6. J. Biol. Chem. 272:32078-32083.

22. Bonecchi, R., et al. 2004. Differential recognition and scavenging of native and truncated macrophage-derived chemokine (macrophage-derived chemokine/CC chemokine ligand 22) by the D6 decoy receptor. J. Immunol. 172:4972-4976.

23. Mantovani, A., Bonecchi, R., and Locati, M. 2006. Tuning inflammation and immunity by chemokine sequestration: decoys and more. Nat. Rev. Immunol. 6:907-918.

24. Martinez de la Torre, Y., et al. 2007. Protection against inflammation- and autoantibody-caused fetal loss by the chemokine decoy receptor D6. Proc. Natl. Acad. Sci. U. S. A. 104:2319-2324.

25. Nibbs, R., Graham, G., and Rot, A. 2003. Chemokines on the move: control by the chemokine “interceptors" Duffy blood group antigen and D6. Semin. Immunol. 15:287-294.

26. Fra, A.M., et al. 2003. Cutting edge: scavenging of inflammatory CC chemokines by the promiscuous putatively silent chemokine receptor D6. J. Immunol. 170:2279-2282.

27. Galliera, E., et al. 2004. beta-Arrestin-dependent constitutive internalization of the human chemokine decoy receptor D6. J. Biol. Chem. 279:25590-25597.

28. Weber, M., et al. 2004. The chemokine receptor D6 constitutively traffics to and from the cell surface to internalize and degrade chemokines. Mol. Biol. Cell. 15:2492-2508.

29. Jamieson, T., et al. 2005. The chemokine receptor D6 limits the inflammatory response in vivo. Nat. Immunol. 6:403-411.

30. Martinez de la Torre, Y., et al. 2005. Increased inflammation in mice deficient for the chemokine decoy receptor D6. Eur. J. Immunol. 35:1342-1346.

31. Whitehead, G.S., et al. 2007. The chemokine receptor D6 has opposing effects on allergic inflammation and airway reactivity. Am. J. Respir. Crit. Care Med. 175:243-249.

32. Woodworth, C.D., et al. 2004. Strain-dependent differences in malignant conversion of mouse skin tumors is an inherent property of the epidermal 
keratinocyte. Carcinogenesis. 25:1771-1778.

33. Mao, J.H., et al. 2004. Mutually exclusive mutations of the Pten and ras pathways in skin tumor progression. Genes Dev. 18:1800-1805.

34. Mao, L., Hong, W.K., and Papadimitrakopoulou, V.A. 2004. Focus on head and neck cancer. Cancer Cell. 5:311-316.

35. Mignogna, M.D., Fedele, S., Lo Russo, L., Lo Muzio, L., and Bucci, E. 2004. Immune activation and chronic inflammation as the cause of malignancy in oral lichen planus: is there any evidence? Oral Oncol. 40:120-130.

36. Reiss, Y., Proudfoot, A.E., Power, C.A., Campbell, J.J., and Butcher, E.C. 2001. CC chemokine receptor (CCR) 4 and the CCR10 ligand cutaneous T cell-attracting chemokine (CTACK) in lymphocyte trafficking to inflamed skin. J. Exp. Med. 194:1541-1547.

37. Homey, B., et al. 2002. CCL27-CCR10 interactions regulate $\mathrm{T}$ cell-mediated skin inflammation. Nat. Med. 8:157-165.

38. Scott, K., and Bradding, P. 2005. Human mast cell chemokines receptors: implications for mast cell tissue localization in asthma. Clin. Exp. Allergy. 35:693-697.

39. Starkey, J.R., Crowle, P.K., and Taubenberger, S. 1988. Mast-cell-deficient W/Wv mice exhibit a decreased rate of tumor angiogenesis. Int. J. Cancer. 42:48-52.

40. Girardi, M., et al. 2002. Resident skin-specific gammadelta $T$ cells provide local, nonredundant regulation of cutaneous inflammation. J. Exp. Med. 195:855-867.

41. Gilfillan, A.M., and Tkaczyk, C. 2006. Integrated signalling pathways for mast-cell activation. Nat. Rev. Immunol. 6:218-230.

42. Miyazaki, D., et al. 2005. Macrophage inflammatory protein-1alpha as a costimulatory signal for mast cell-mediated immediate hypersensitivity reactions. J. Clin. Invest. 115:434-442. doi:10.1172/ JCI200518452.

43. Sparmann, A., and Bar-Sagi, D. 2004. Ras-induced interleukin-8 expression plays a critical role in tumor growth and angiogenesis. Cancer Cell. 6:447-458.

44. Strieter, R.M., Burdick, M.D., Gomperts, B.N., Belperio, J.A., and Keane, M.P. 2005. CXC chemo- kines in angiogenesis. Cytokine Growth Factor Rev. 16:593-609.

45. Wang, H.W., et al. 2004. Kaposi sarcoma herpesvirus-induced cellular reprogramming contributes to the lymphatic endothelial gene expression in Kaposi sarcoma. Nat. Genet. 36:687-693.

46. Vasioukhin, V., Degenstein, L., Wise, B., and Fuchs, E. 1999. The magical touch: genome targeting in epidermal stem cells induced by tamoxifen application to mouse skin. Proc. Natl. Acad. Sci. U. S. A. 96:8551-8556.

47. Yao, D., et al. 2006. PTEN loss promotes rasHamediated papillomatogenesis via dual up-regulation of AKT activity and cell cycle deregulation but malignant conversion proceeds via PTEN-associated pathways. Cancer Res. 66:1302-1312.

48. Kemp, C.J., Donehower, L.A., Bradley, A., and Balmain, A. 1993. Reduction of p53 gene dosage does not increase initiation or promotion but enhances malignant progression of chemically induced skin tumors. Cell. 74:813-822.

49. Hunter, K.D., et al. 2006. Divergent routes to oral cancer. Cancer Res. 66:7405-7413. 